\title{
KNOT INVARIANTS ARISING FROM HOMOLOGICAL OPERATIONS ON KHOVANOV HOMOLOGY
}

\author{
KRZYSZTOF K. PUTYRA AND ALEXANDER N. SHUMAKOVITCH
}

\begin{abstract}
We construct an algebra of non-trivial homological operations on Khovanov homology with coefficients in $\mathbb{Z}_{2}$ generated by two Bockstein operations. We use the unified Khovanov homology theory developed by the first author to lift this algebra to integral Khovanov homology. We conjecture that these two algebras are infinite and present evidence in support of our conjectures. Finally, we list examples of knots that have the same even and odd Khovanov homology, but different actions of these homological operations. This confirms that the unified theory is a finer knot invariant than the even and odd Khovanov homology combined. The case of reduced Khovanov homology is also considered.
\end{abstract}

\section{INTRODUCTION}

Throughout this paper, $L$ will denote a link in $\mathbb{R}^{3}$ and $D$ its planar diagram. There are two integral versions of $\mathfrak{s l}_{2}$ link homology of $L$ : the ordinary Khovanov homology $\mathcal{H}_{e}(L)$ [Kh99], which we refer to is this paper as even, and the odd Khovanov homology $\mathcal{H}_{o}(L)$ [ORS13]. Despite being different over integers [Sh11], even and odd Khovanov homology theories agree modulo 2. We denote the resulting homology by $\mathcal{H}_{\mathbb{Z}_{2}}(L)$. Differentials in the even and odd Khovanov chain complexes induce two Bockstein connecting homomorphisms on $\mathcal{H}_{\mathbb{Z}_{2}}(L)$ that correspond to the short exact sequence of coefficients $0 \longrightarrow \mathbb{Z}_{2} \longrightarrow \mathbb{Z}_{4} \longrightarrow \mathbb{Z}_{2} \longrightarrow 0$. We denote them by $\beta_{e}$ and $\beta_{o}$ respectively:

$$
\beta_{e}, \beta_{o}: \mathcal{H}_{\mathbb{Z}_{2}}^{i}(L) \longrightarrow \mathcal{H}_{\mathbb{Z}_{2}}^{i+1}(L) .
$$

Ranks of each of $\beta_{e}$ and $\beta_{o}$ can be easily recovered from the integral homology: they are equal to the number of invariant factors isomorphic to $\mathbb{Z}_{2}$ in the corresponding homology group, see [Ha10, Proposition 3E.3]. On the other hand, $\beta_{e}$ and $\beta_{o}$ are as algebraically independent as possible: they not only do not commute, but all their alternating compositions are nontrivial. More precisely, let $\beta:=\beta_{e}+\beta_{o}$. Since $\beta_{e}^{2}=\beta_{o}^{2}=0$, we have that $\beta \beta_{e}=\beta_{o} \beta_{e}$ and $\beta \beta_{e}=\beta_{e} \beta_{o}$. Hence, the only nontrivial compositions of Bockstein homomorphisms of length $n$ are $\beta^{n-1} \beta_{e}$ and $\beta^{n-1} \beta_{o}$, whereas $\beta^{n}$ is their sum.

The even Khovanov homology is multiplicative with respect to disjoint unions of links, $\mathcal{H}_{e}\left(L \sqcup L^{\prime}\right) \cong \mathcal{H}_{e}(L) \hat{\otimes} \mathcal{H}_{e}\left(L^{\prime}\right)[$ Kh99] (here $\hat{\otimes}$ stands for the derived tensor product), which

KKP is supported by the NCCR SwissMAP founded by the Swiss National Science Foundation.

A.Sh. is partially supported by a Simons Collaboration Grant for Mathematicians \#279867. 
implies primitivity of the even Bockstein homomorphism $\beta_{e}$. The first author has recently proved the multiplicativity of the odd homology [Pu14], which implies primitivity of $\beta_{o}$. Similar formulas hold for connected sums of knots, in which case one considers a derived tensor product over the algebra assigned to a circle [Kh04, Pu14]. This allowed us to construct in Example 3.7 a knot with 20 crossings for which the alternating compositions of four Bockstein homomorphisms are nontrivial.

Conjecture 3.8. The operations $\beta^{n}$, $\beta^{n-1} \beta_{e}$, and $\beta^{n-1} \beta_{o}$ are nonzero and pairwise different.

The two Bockstein homomorphisms generate three degree 2 operations: $\beta_{e} \beta_{o}, \beta_{o} \beta_{e}$, and the commutator $\beta^{2}=\beta_{e} \beta_{o}+\beta_{o} \beta_{e}$. It appears that none of them is the second Steenrod square $S q^{2}$ as defined in [LS12], because the ranks do not match. Moreover $\beta^{2}$ is primitive, while $S q^{2}$ is not (see [Ha10, Section 4.L]).

Corollary 1.1. The degree 2 operations $\beta_{e} \beta_{o}, \beta_{o} \beta_{e}$, and $\beta^{2}$ are different from the second Steenrod square $S q^{2}$.

Another surprising fact is the existence of integral lifts of both Bockstein operations. They are constructed using a link homology $\mathcal{H}_{\xi}(L)$ that is defined over a ring $\mathbb{Z}_{\xi}:=\mathbb{Z}[\xi] /\left(\xi^{2}-1\right)$ and unifies the even and odd Khovanov homology theories. $\mathcal{H}_{e}(L)$ and $\mathcal{H}_{o}(L)$ can be recovered from $\mathcal{H}_{\xi}(L)$ by taking coefficients in certain modules over $\mathbb{Z}_{\xi}[\mathrm{Pu} 13]$. Namely, there are isomorphisms of graded abelian groups

$$
\mathcal{H}_{e}(L) \cong \mathcal{H}_{\xi}\left(L ; \mathbb{Z}_{e}\right) \quad \text { and } \quad \mathcal{H}_{o}(L) \cong \mathcal{H}_{\xi}\left(L ; \mathbb{Z}_{o}\right)
$$

where $\mathbb{Z}_{e}:=\mathbb{Z}_{\xi} /(\xi-1)$ and $\mathbb{Z}_{o}:=\mathbb{Z}_{\xi} /(\xi+1)$ are $\mathbb{Z}_{\xi}$-modules on which $\xi$ acts as identity or negation respectively. Both $\mathbb{Z}_{e}$ and $\mathbb{Z}_{o}$ are isomorphic to $\mathbb{Z}$ as abelian groups.

It is not immediately clear whether $\mathcal{H}_{\xi}(L)$ is a stronger invariant than $\mathcal{H}_{e}(L)$ and $\mathcal{H}_{o}(L)$ together. The authors are not aware of any software package that can compute the unified homology explicitly. This might be due to the complexity of classification of modules over the ring $\mathbb{Z}_{\xi}$. The results of this paper indicate that $\mathcal{H}_{\xi}(L)$ is actually a finer invariant than the even and odd homology combined.

Let $D$ be a diagram of a link $L$ in $\mathbb{R}^{3}$. Denote by $\mathcal{C}_{e}(D), \mathcal{C}_{o}(D)$, and $\mathcal{C}_{\xi}(D)$ the Khovanov chain complexes for the even, odd, and unified homology, respectively. We recall in Section 2 that $\mathcal{C}_{\xi}(D)$ can be considered as an extension of complexes $\mathcal{C}_{e}(D)$ and $\mathcal{C}_{o}(D)$ in two different ways. The two short exact sequences

$$
\begin{aligned}
& 0 \longrightarrow \mathcal{C}_{o}(D) \longrightarrow \mathcal{C}_{\xi}(D) \longrightarrow \mathcal{C}_{e}(D) \longrightarrow 0 \\
& 0 \longrightarrow \mathcal{C}_{e}(D) \longrightarrow \mathcal{C}_{\xi}(D) \longrightarrow \mathcal{C}_{o}(D) \longrightarrow 0
\end{aligned}
$$

induce connecting homomorphisms $\varphi_{e o}: \mathcal{H}_{e}^{i}(L) \rightarrow \mathcal{H}_{o}^{i+1}(L)$ and $\varphi_{o e}: \mathcal{H}_{o}^{i}(L) \rightarrow \mathcal{H}_{e}^{i+1}(L)$. In Section 4 we show that $\varphi_{e o}$ and $\varphi_{o e}$ are, in fact, Bockstein homomorphisms corresponding to 
certain short exact sequences of $\mathbb{Z}_{\xi}$-modules. Moreover, they are integral lifts of the Bockstein homomorphisms $\beta_{o}$ and $\beta_{e}$, respectively.

Proposition 4.1. Given a link $L$ there are commuting squares
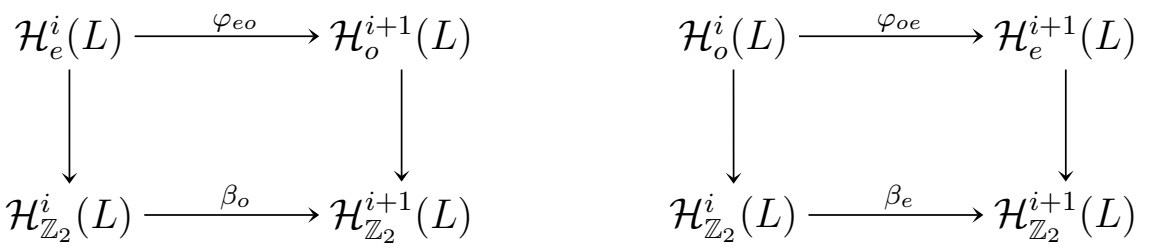

Similarly to the Bockstein operations over $\mathbb{Z}_{2}$, we expect that the alternating compositions of the integral operations do not vanish, see Conjecture 4.3.

Computer-based calculations reveal pairs of knots that have the same homology groups (both odd and even), but different actions of the integral operation, see Section 6. This implies that the unified link homology $\mathcal{H}_{\xi}(L)$ is a stronger invariant than $\mathcal{H}_{e}(L) \oplus \mathcal{H}_{o}(L)$. In particular, $\mathcal{C}_{\xi}(L)$ is a nontrivial extension of $\mathcal{C}_{e}(L)$ and $\mathcal{C}_{o}(L)$.

Remark 1.2. Most of the constructions described in this paper can be applied to arbitrary complexes over the ring $\mathbb{Z}_{\xi}$, not necessarily those arising from links. Indeed, with every such a complex $C$ we can associate its 'even' and 'odd' integral versions, $C_{e}:=C \otimes \mathbb{Z}_{e}$ and $C_{o}:=C \otimes \mathbb{Z}_{o}$, that have the same reductions modulo two $C_{\mathbb{Z}_{2}}:=C_{e} \otimes \mathbb{Z}_{2} \cong C_{o} \otimes \mathbb{Z}_{2}$. The differentials in $C_{e}$ and $C_{o}$ induce two Bockstein homomorphisms on $\mathcal{H}\left(C_{\mathbb{Z}_{2}} ; \mathbb{Z}_{2}\right)$, each admitting an integral lift.

Outline. This paper is organized as follows. We begin with a pullback description of the ring $\mathbb{Z}_{\xi}$ and the unified Khovanov homology $\mathcal{H}_{\xi}(L)$. It provides a neat way to see the even and odd Khovanov complexes as subcomplexes and quotient complexes of $\mathcal{C}_{\xi}(L)$ at the same time. Section 3 describes the construction of Bockstein operations for homology with coefficients in $\mathbb{Z}_{2}$. We present examples of knots for which compositions of up to four Bocksteins are nontrivial to support Conjecture 3.8. Integral Bockstein operations and the integral analog of Conjecture 3.8 are treated in Section 4. Section 5 contains a brief discussion of the case of the reduced Khovanov homology. The results of computer-based calculation are presented in Section 6.

Summary of notation. In this paper, for every diagram $D$ of a link $L$ we assign a number of different chain complexes and their homology. The notation used is listed in Table 1. We also define several homological operations on different versions of Khovanov homology. For convenience, we list pages on which they are introduced in Table 2.

Acknowledgements. We are grateful to Mikhail Khovanov for numerous stimulating discussions. The first author is also thankful to Stefan Friedl for his insightful remarks during the SwissKnots 2011 conference. 


\begin{tabular}{l|cccc}
\hline & even & odd & unified & $\begin{array}{c}\text { reduction } \\
\text { modulo 2 }\end{array}$ \\
\hline chain complex & $\mathcal{C}_{e}(D)$ & $\mathcal{C}_{o}(D)$ & $\mathcal{C}_{\xi}(D)$ & $\mathcal{C}_{\mathbb{Z}_{2}}(D)$ \\
differential & $d_{e}$ & $d_{o}$ & $d_{\xi}$ & $d$ \\
homology & $\mathcal{H}_{e}(L)$ & $\mathcal{H}_{o}(L)$ & $\mathcal{H}_{\xi}(L)$ & $\mathcal{H}_{\mathbb{Z}_{2}}(L)$ \\
\hline
\end{tabular}

TABLE 1. Notations for different versions of the Khovanov chain complexes and their homology for a diagram $D$ of a link $L$.

$$
\begin{array}{rlr}
\beta_{e}: \mathcal{H}_{\mathbb{Z}_{2}}^{i}(L) \longrightarrow \mathcal{H}_{\mathbb{Z}_{2}}^{i+1}(L) & \text { even Bockstein homomorphism } & \text { p. } 5 \\
\beta_{o}: \mathcal{H}_{\mathbb{Z}_{2}}^{i}(L) \longrightarrow \mathcal{H}_{\mathbb{Z}_{2}}^{i+1}(L) & \text { odd Bockstein homomorphism } & \text { p. } 5 \\
\beta: \mathcal{H}_{\mathbb{Z}_{2}}^{i}(L) \longrightarrow \mathcal{H}_{\mathbb{Z}_{2}}^{i+1}(L) & \text { mixed Bockstein homomorphism } & \text { p. } 5 \\
\varphi_{o e}: \mathcal{H}_{o}^{i}(L) \longrightarrow \mathcal{H}_{e}^{i+1}(L) & \text { integral even Bockstein homomorphism } & \text { p. } 9 \\
\varphi_{e o}: \mathcal{H}_{e}^{i}(L) \longrightarrow \mathcal{H}_{o}^{i+1}(L) & \text { integral odd Bockstein homomorphism } & \text { p. } 9 \\
\theta_{e}: \mathcal{H}_{e}^{i}(L) \longrightarrow \mathcal{H}_{e}^{i+2}(L) & \text { integral even degree 2 operation } & \text { p. } 15 \\
\theta_{o}: \mathcal{H}_{o}^{i}(L) \longrightarrow \mathcal{H}_{o}^{i+2}(L) & \text { integral odd degree 2 operation } & \text { p. } 15
\end{array}
$$

TABLE 2. List of homological operations together with pages on which they are introduced.

\section{A PULLBACK DESCRIPTION OF THE UNIFIED HOMOLOGY}

As mentioned in the introduction, $\mathbb{Z}_{\xi}=\mathbb{Z}[\xi] /\left(\xi^{2}-1\right)$ is the universal ring of coefficients in our framework. The rings $\mathbb{Z}_{e}$ and $\mathbb{Z}_{o}$ arise as quotients of $\mathbb{Z}_{\xi}$, and both project to $\mathbb{Z}_{2}$ in a unique way. Notice, that $\mathbb{Z}_{2}$ admits a unique $\mathbb{Z}_{\xi}$-module structure, as $\xi$ is invertible. Thence we obtain a commuting square diagram shown to the right. In fact, it is a pullback square in the category of rings.

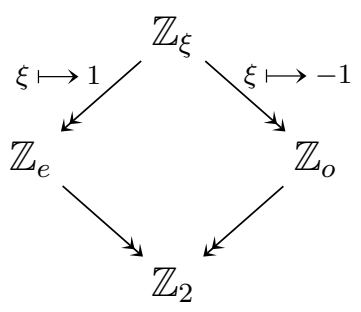

Lemma 2.1. There is an isomorphism of rings $\mathbb{Z}_{\xi} \cong\left\{(a, b) \in \mathbb{Z}^{2} \mid a \equiv b \bmod 2\right\}$, such that the projections on the first and on the second factors are exactly $\mathbb{Z}_{e}$ and $\mathbb{Z}_{o}$.

Proof. The desired isomorphism maps 1 to $(1,1)$ and $\xi$ to $(1,-1)$. This map is injective, since $(a+b, a-b)=(0,0)$ implies $a=b=0$, and surjective, as $(a, b)$ with $a \equiv b \bmod 2$ is an image of $\frac{a+b}{2}+\frac{a-b}{2} \xi$. To finish the proof, notice that the action of $\xi$ preserves the first factor, but negates the second. 
Corollary 2.2. Given a link diagram $D$, the chain complex $\mathcal{C}_{\xi}(D)$ is a pullback of the even and odd Khovanov complexes over their reductions modulo 2. In particular, it is a subcomplex of the direct $\operatorname{sum} \mathcal{C}_{e}(D) \oplus \mathcal{C}_{o}(D)$.

Proof. The chain complex $\mathcal{C}_{\xi}(D)$ is a sequence of free $\mathbb{Z}_{\xi}$-modules, so that the functor $M \longmapsto \mathcal{C}_{\xi}(D) \otimes M$, where $M$ runs over $\mathbb{Z}_{\xi}$-modules, is exact. In particular, it preserves pullbacks.

According to the corollary above, we can regard $\mathcal{H}_{\xi}(L)$ as a derived pullback of the diagram

$$
\mathcal{H}_{e}(L) \longrightarrow \mathcal{H}_{\mathbb{Z}_{2}}(L) \longleftarrow \mathcal{H}_{o}(L) .
$$

Furthermore, the kernel of the projection $\mathcal{C}_{\xi}(D) \longrightarrow \mathcal{C}_{e}(D)$ is the subcomplex of $\mathcal{C}_{o}(D)$ formed by elements divisible by 2 , which is isomorphic to the odd Khovanov complex. Likewise, the kernel of $\mathcal{C}_{\xi}(D) \longrightarrow \mathcal{C}_{o}(D)$ is isomorphic to $\mathcal{C}_{e}(D)$. Hence, there are short exact sequences

$$
\begin{aligned}
& 0 \longrightarrow \mathcal{C}_{o}(D) \longrightarrow \mathcal{C}_{\xi}(D) \longrightarrow \mathcal{C}_{e}(D) \longrightarrow 0, \text { and } \\
& 0 \longrightarrow \mathcal{C}_{e}(D) \longrightarrow \mathcal{C}_{\xi}(D) \longrightarrow \mathcal{C}_{o}(D) \longrightarrow 0,
\end{aligned}
$$

so that $\mathcal{C}_{\xi}(D)$ is an extension between the two chain complexes. We shall show in the subsequent sections that its homology is a stronger invariant than both even and odd homology together. However, the difference is very subtle.

Proposition 2.3. Let $f: \mathcal{C}_{e}(L) \longrightarrow \mathcal{C}_{e}\left(L^{\prime}\right)$ and $g: \mathcal{C}_{o}(L) \longrightarrow \mathcal{C}_{o}\left(L^{\prime}\right)$ be quasi-isomorphisms ${ }^{1}$ that agree modulo 2. Then $\mathcal{H}_{\xi}(L) \cong \mathcal{H}_{\xi}\left(L^{\prime}\right)$.

Proof. The pullback $(f, g)$ of the chain maps $f$ and $g$ is the desired quasi-isomorphism, which follows from the 5-lemma applied to the exact sequence (6).

\section{Bockstein operations in Khovanov homology}

Since the odd and even differentials agree modulo 2, there are at least three Bockstein operations on $\mathcal{H}_{\mathbb{Z}_{2}}(L)$ :

1. the even Bockstein, $\beta_{e}: \mathcal{H}_{\mathbb{Z}_{2}}^{i}(L) \longrightarrow \mathcal{H}_{\mathbb{Z}_{2}}^{i+1}(L), \beta_{e}[x]=\left[\frac{1}{2} d_{e} x\right]$,

2. the odd Bockstein, $\beta_{o}: \mathcal{H}_{\mathbb{Z}_{2}}^{i}(L) \longrightarrow \mathcal{H}_{\mathbb{Z}_{2}}^{i+1}(L), \beta_{o}[x]=\left[\frac{1}{2} d_{o} x\right]$, and

3. the mixed Bockstein, $\beta:=\beta_{e}+\beta_{o}$.

The last one arises from the short exact sequence of coefficients

$$
0 \longrightarrow \mathbb{Z}_{2} \longrightarrow \mathbb{Z}_{2}[\xi] /\left(\xi^{2}-1\right) \longrightarrow \mathbb{Z}_{2} \longrightarrow 0
$$

where the inclusion of $\mathbb{Z}_{2}$ maps 1 into $1+\xi$. Indeed, from the pullback description of $\mathcal{C}_{\xi}(L)$ we have $d_{\xi}=\frac{1}{2}\left(d_{e}+d_{o}\right)+\frac{\xi}{2}\left(d_{e}-d_{o}\right)=\frac{1+\xi}{2}\left(d_{e}+d_{o}\right)$, so that $\beta[x]=\left[\frac{1}{2} d_{\xi} x\right]=\left[\frac{1}{2} d_{e} x+\frac{1}{2} d_{o} x\right]$.

\footnotetext{
${ }^{1}$ A chain map is a quasi-isomorphism if it induces an isomorphism on homology.
} 
To see that there is nothing more, use the free resolution

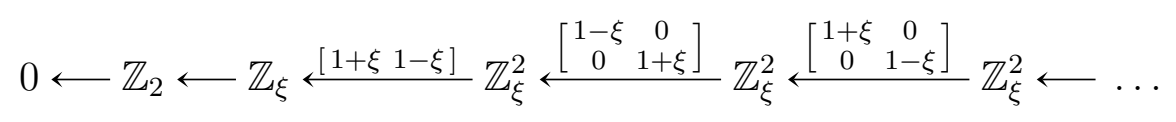

to compute $\operatorname{Ext}_{\mathbb{Z}_{\xi}}^{1}\left(\mathbb{Z}_{2}, \mathbb{Z}_{2}\right) \cong \mathbb{Z}_{2} \oplus \mathbb{Z}_{2}$. In particular, the sum of any two of the three Bocksteins results in the third.

Choose a chain complex $C$ with a differential of degree 1. Due to the Universal Coefficient Theorem, the homology of $C$ with coefficients in $\mathbb{Z}_{2}$ is given as the direct $\operatorname{sum} H^{i}\left(C, \mathbb{Z}_{2}\right) \cong$ $H^{i}(C) \otimes \mathbb{Z}_{2} \oplus \operatorname{Tor}\left(H^{i+1}(C), \mathbb{Z}_{2}\right)$. Let $u \in \operatorname{Tor}\left(H^{i+1}(C), \mathbb{Z}_{2}\right)$ be an element of order 2 that arises from an invariant factor of $H^{i+1}(C)$ that is isomorphic to $\mathbb{Z}_{2}$. Then the Bockstein homomorphism on $H^{i}\left(C, \mathbb{Z}_{2}\right)$ pairs $u$ with the modulo 2 reduction of the homology class that $u$ comes from. It sends every element of $H^{i}\left(C, \mathbb{Z}_{2}\right)$ that does not arise in such a way to zero, see [Ha10, Proposition 3E.3].

Example 3.1. The odd Khovanov homology of alternating links has no torsion [ORS13], so that the odd Bockstein is trivial. On the other hand, the even Khovanov homology of any torus knot $T_{2, n}$ contains a $\mathbb{Z}_{2}$ summand [Kh99], and the even Bockstein does not vanish.

The following result follows directly from the definition of the operations.

Proposition 3.2. The odd and even Bockstein homomorphisms are differentials, i.e. $\beta_{e}^{2}=$ $\beta_{o}^{2}=0$ and $\beta^{2}=\left[\beta_{e}, \beta_{o}\right]$ is their commutator.

Thence, there are at most three nontrivial operations in each degree that are generated by the Bocksteins: the alternating compositions $\underbrace{\cdots \beta_{o} \beta_{e}}_{n}=\beta^{n-1} \beta_{e}$ and $\underbrace{\cdots \beta_{e} \beta_{o}}_{n}=\beta^{n-1} \beta_{o}$,
together with their sum $\beta^{n}$.

According to Example 3.1 the even and odd Bockstein homomorphisms are linearly independent. The next two examples show the same for $\beta_{o} \beta_{e}$ and $\beta_{e} \beta_{o}$.

Example 3.3. Consider the torus knot $T_{3,4}$, labeled as $8_{19}$ in the Rolfsen's table [Ro76]. Its even and odd Khovanov homology are presented in the top two tables of Figure 1. An analysis of positions of $\mathbb{Z}_{2}$ summands results in the bottom table in Figure 1, where the horizontal arrows illustrate nontrivial contributions to the Bockstein homomorphisms. Notice that the composition $\beta_{o} \beta_{e}$ does not vanish on the generator $u$ of $\mathcal{H}_{\mathbb{Z}_{2}}^{2,11}\left(8_{19}\right) \cong \mathbb{Z}_{2}$ and $\beta^{2}(u) \neq 0$. Therefore, the two Bockstein homomorphisms do not commute with each other.

Example 3.4. The torus knot $T_{3,5}$, labeled as $10_{124}$ in the Rolfsen's table [Ro76], admits a class with the opposite property: $\beta_{e} \beta_{o}$ does not not vanish on the generator $u$ of $\mathcal{H}_{\mathbb{Z}_{2}}^{5,19}\left(10_{124}\right) \cong \mathbb{Z}_{2}$, see Figure 2 . 


\begin{tabular}{||r||l|l|l|l|l|l||}
\hline \hline $\mathcal{H}_{e}$ & 0 & 1 & 2 & 3 & 4 & 5 \\
\hline \hline 17 & & & & & & $\mathbb{Z}$ \\
\hline 15 & & & & & & $\mathbb{Z}$ \\
\hline 13 & & & & $\mathbb{Z}$ & $\mathbb{Z}$ & \\
\hline 11 & & & & $\mathbb{Z}_{2}$ & $\mathbb{Z}$ & \\
\hline 9 & & & $\mathbb{Z}$ & & & \\
\hline 7 & $\mathbb{Z}$ & & & & & \\
\hline 5 & $\mathbb{Z}$ & & & & & \\
\hline \hline
\end{tabular}

\begin{tabular}{||r||c|c|c|c|c|c||}
\hline \hline $\mathcal{H}_{o}$ & 0 & 1 & 2 & 3 & 4 & 5 \\
\hline \hline 17 & & & & & & $\mathbb{Z}$ \\
\hline 15 & & & & & & $\mathbb{Z} \oplus \mathbb{Z}_{3}$ \\
\hline 13 & & & & & $\mathbb{Z}_{2}$ & $\mathbb{Z}_{3}$ \\
\hline 11 & & & $\mathbb{Z}$ & & $\mathbb{Z}_{2}$ & \\
\hline 9 & & & $\mathbb{Z}$ & & & \\
\hline 7 & $\mathbb{Z}$ & & & & & \\
\hline 5 & $\mathbb{Z}$ & & & & & \\
\hline \hline
\end{tabular}

\begin{tabular}{||r||l|l|l|l|l|l||}
\hline \hline $\mathcal{H}_{\mathbb{Z}_{2}}$ & 0 & 1 & 2 & 3 & 4 & 5 \\
\hline \hline 17 & & & & & & $\mathbb{Z}_{2}$ \\
\hline 15 & & & & & & $\mathbb{Z}_{2}$ \\
\hline 13 & & & & $\mathbb{Z}_{2} \stackrel{\beta_{0}}{\rightarrow} \mathbb{Z}_{2}$ & \\
\hline 11 & & & $\mathbb{Z}_{2} \stackrel{\beta_{e}}{\longrightarrow} \mathbb{Z}_{2} \stackrel{\beta_{o}}{\longrightarrow} \mathbb{Z}_{2}$ & \\
\hline 9 & & & $\mathbb{Z}_{2}$ & & & \\
\hline 7 & $\mathbb{Z}_{2}$ & & & & & \\
\hline 5 & $\mathbb{Z}_{2}$ & & & & & \\
\hline \hline
\end{tabular}

Figure 1. Even and odd Khovanov homology as well as Bockstein homomorphisms on the Khovanov homology over $\mathbb{Z}_{2}$ for the knot $8_{19}$.

The even Khovanov homology is multiplicative with respect to disjoint union and connected sum of links:

$$
\begin{aligned}
& \mathcal{C}_{e}\left(D \sqcup D^{\prime}\right) \cong \mathcal{C}_{e}(D) \underset{\mathbb{Z}_{2}}{\otimes} \mathcal{C}_{e}\left(D^{\prime}\right), \\
& \mathcal{C}_{e}\left(D \# D^{\prime}\right) \cong \mathcal{C}_{e}(D) \underset{A}{\otimes} \mathcal{C}_{e}\left(D^{\prime}\right),
\end{aligned}
$$

where $A=\mathbb{Z} v_{+} \oplus \mathbb{Z} v_{-}$is the Frobenius algebra associated to a circle [Kh99, Kh04]. In the latter formula, we regard $\mathcal{C}_{e}(D)$ as an $A$-module with the action of $A$ induced by the cobordism that merges a circle to the link diagram at the place where the connected sum is performed, see Figure 3.

The same formulas were recently proved for the odd Khovanov chain complexes [Pu14]. Since $\mathbb{Z}_{2}$ is a field, the Künneth formula identifies the homology of the disjoint union or connected sum as tensor products of homology:

$$
\mathcal{H}_{\mathbb{Z}_{2}}\left(L \sqcup L^{\prime}\right) \cong \mathcal{H}_{\mathbb{Z}_{2}}(L) \underset{\mathbb{Z}_{2}}{\otimes} \mathcal{H}_{\mathbb{Z}_{2}}\left(L^{\prime}\right),
$$




\begin{tabular}{||r||l|l|l|l|l|l|l|l||}
\hline \hline $\mathcal{H}_{e}$ & 0 & 1 & 2 & 3 & 4 & 5 & 6 & 7 \\
\hline \hline 21 & & & & & & & & $\mathbb{Z}$ \\
\hline 19 & & & & & & $\mathbb{Z}$ & & $\mathbb{Z}_{2}$ \\
\hline 17 & & & & & & $\mathbb{Z}$ & $\mathbb{Z}$ & \\
\hline 15 & & & & $\mathbb{Z}$ & $\mathbb{Z}$ & & & \\
\hline 13 & & & & $\mathbb{Z}_{2}$ & $\mathbb{Z}$ & & & \\
\hline 11 & & & $\mathbb{Z}$ & & & & & \\
\hline 9 & $\mathbb{Z}$ & & & & & & & \\
\hline 7 & $\mathbb{Z}$ & & & & & & & \\
\hline \hline
\end{tabular}

\begin{tabular}{||r||l|l|l|l|l|l|l|l||}
\hline \hline $\mathcal{H}_{o}$ & 0 & 1 & 2 & 3 & 4 & 5 & 6 & 7 \\
\hline \hline 21 & & & & & & & & $\mathbb{Z}$ \\
\hline 19 & & & & & & & $\mathbb{Z}_{2}$ & $\mathbb{Z}$ \\
\hline 17 & & & & & & $\mathbb{Z}_{3}$ & $\mathbb{Z}_{2}$ & \\
\hline 15 & & & & & $\mathbb{Z}_{2}$ & $\mathbb{Z}_{3}$ & & \\
\hline 13 & & & $\mathbb{Z}$ & & $\mathbb{Z}_{2}$ & & & \\
\hline 11 & & & $\mathbb{Z}$ & & & & & \\
\hline 9 & $\mathbb{Z}$ & & & & & & & \\
\hline 7 & $\mathbb{Z}$ & & & & & & & \\
\hline \hline
\end{tabular}

\begin{tabular}{|c|c|c|c|c|c|c|c|c|}
\hline $\mathcal{H}_{\mathbb{Z}_{2}}$ & 0 & 1 & 2 & 3 & 4 & 5 & 6 & 7 \\
\hline 21 & & & & & & & & $\mathbb{Z}_{2}$ \\
\hline 19 & & & & & & & $\mathbb{Z}_{2}$ & $\rightarrow \mathbb{Z}_{2}$ \\
\hline 17 & & & & & & $\mathbb{Z}_{2}$ & $\rightarrow \mathbb{Z}_{2}$ & \\
\hline 15 & & & & \multicolumn{2}{|c|}{$\mathbb{Z}_{2} \stackrel{\beta_{0}}{\mid} \mathbb{Z}_{2}$} & & & \\
\hline 13 & & & \multicolumn{3}{|c|}{$\mathbb{Z}_{2} \stackrel{\beta_{e}}{\mid} \mathbb{Z}_{2} \stackrel{\beta_{o}}{\mid} \mathbb{Z}_{2}$} & & & \\
\hline 11 & & & $\mathbb{Z}_{2}$ & & & & & \\
\hline 9 & $\mathbb{Z}_{2}$ & & & & & & & \\
\hline 7 & $\mathbb{Z}_{2}$ & & & & & & & \\
\hline
\end{tabular}

Figure 2. Even and odd Khovanov homology as well as Bockstein homomorphisms on the Khovanov homology over $\mathbb{Z}_{2}$ for the knot $10_{124}$.

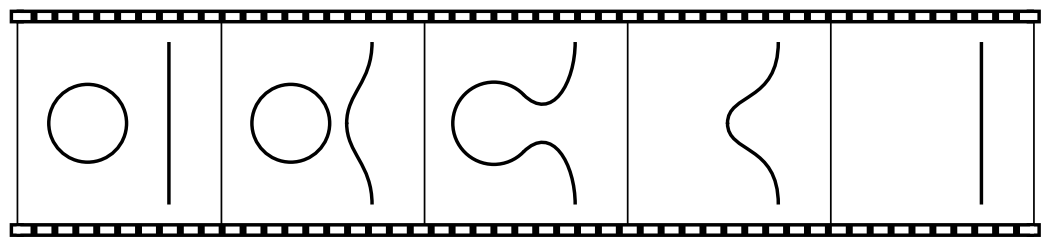

Figure 3. Sections of the cobordism inducing an action of $A$ on the Khovanov chain complex $\mathcal{C}_{e}(D)$.

$$
\mathcal{H}_{\mathbb{Z}_{2}}\left(L \# L^{\prime}\right) \cong \mathcal{H}_{\mathbb{Z}_{2}}(L) \underset{A}{\otimes} \mathcal{H}_{\mathbb{Z}_{2}}\left(L^{\prime}\right)
$$

Definition 3.5. A homological operation $\theta_{L}: \mathcal{H}_{\mathbb{Z}_{2}}^{i}(L) \longrightarrow \mathcal{H}_{\mathbb{Z}_{2}}^{i+d}(L)$ of degree $d$ is said to be

- $\sqcup$-primitive if $\theta_{L \sqcup L^{\prime}}=\theta_{L} \underset{\mathbb{Z}_{2}}{\otimes} \mathrm{id}+\mathrm{id} \underset{\mathbb{Z}_{2}}{\otimes} \theta_{L^{\prime}}$ and

- \#-primitive if $\theta_{L \# L}=\theta_{L} \underset{A}{\otimes} \mathrm{id}+\mathrm{id} \underset{A}{\otimes_{L^{\prime}}}$ 
for every two links $L$ and $L^{\prime}$.

Corollary 3.6. All three Bockstein operations are $\sqcup$-primitive and \#-primitive.

This allows us to construct knots with nontrivial compositions of four Bockstein homomorphisms.

Example 3.7. Consider the generators $u \in \mathcal{H}_{\mathbb{Z}_{2}}^{2,13}\left(10_{124}\right)$ and $u^{\prime} \in \mathcal{H}_{\mathbb{Z}_{2}}^{5,19}\left(10_{124}\right)$. Then $\beta_{o}(u)=$ $0, \beta_{e}\left(u^{\prime}\right)=0, \beta_{o} \beta_{e}(u) \neq 0$, and $\beta_{e} \beta_{o}\left(u^{\prime}\right) \neq 0$ (see Fig. 2). It is now straightforward to verify that $u \otimes u^{\prime} \in \mathcal{H}_{\mathbb{Z}_{2}}^{7,32}\left(10_{124} \# 10_{124}\right)$ satisfies

$$
\beta_{o} \beta_{e} \beta_{o} \beta_{e}\left(u \otimes u^{\prime}\right)=\beta_{e} \beta_{o} \beta_{e} \beta_{o}\left(u \otimes u^{\prime}\right)=\beta_{o} \beta_{e}(u) \otimes \beta_{e} \beta_{o}\left(u^{\prime}\right) \neq 0 .
$$

In particular, both compositions are nontrivial, but $\beta^{4}\left(u \otimes u^{\prime}\right)=0$.

The vanishing of $\beta^{4}$ above is not surprising - it is a well-known fact that given a primitive operation $\theta$, each power $\theta^{2^{r}}$ is also primitive. Indeed, one first computes

$$
\theta^{n}(x \otimes y)=\sum_{k=0}^{n}\left(\begin{array}{l}
n \\
k
\end{array}\right) \theta^{n-k}(x) \otimes \theta^{k}(y)
$$

and then checks that $\left(\begin{array}{c}2^{r} \\ k\end{array}\right)$ is even for all values of $k$ except $k=0$ and $k=2^{r}$. In particular, $\beta^{2^{r}}$ vanishes on the homology of $K_{1} \# K_{2}$ if it vanishes on homology of both $K_{1}$ and $K_{2}$. A quick look at Figure 2 reveals that already $\beta^{3}=0$ for the knot $10_{124}$.

According to the discussion after Proposition 3.2, the existence of nontrivial compositions of length bigger than $\ell$ implies that $\beta^{\ell} \neq 0$. Hence, it is important to find knots for which higher powers of $\beta$ do not vanish.

The torus knot $T_{5,6}$ admits a class $u \in \mathcal{H}_{\mathbb{Z}_{2}}^{5,31}\left(T_{5,6}\right)$, such that $\beta_{e}(u)=0$ but $\beta_{o} \beta_{e} \beta_{o}(u) \neq 0$. Unfortunately, larger knots are beyond the capabilities of our computer program [Sh $\infty$ ], so that we did not succeed in finding a knot for which $\beta^{4} \neq 0$. Nonetheless, we expect that there are examples of knots for which higher powers of $\beta$ are nonzero.

Conjecture 3.8. The operation $\beta^{n}$ does not vanish for the torus $k n o t T_{2 n-1,2 n}$. In particular, for every $n \in \mathbb{N}$ there exists a knot $K_{n}$ such that the operations $\beta^{n}, \beta^{n-1} \beta_{e}$, and $\beta^{n-1} \beta_{o}$ on $\mathcal{H}_{\mathbb{Z}_{2}}\left(K_{n}\right)$ are nonzero and pairwise different.

\section{INTEGRAL LIFTS}

As explained in Section 2, $\mathcal{H}_{\xi}(L)$ can be regarded as an extension of both even and odd Khovanov homology theories. This leads to homological operations between integral Khovanov homologies of a link:

1. $\varphi_{e o}: \mathcal{H}_{e}^{i}(L) \longrightarrow \mathcal{H}_{o}^{i+1}(L)$, the connecting homomorphism for the sequence (6) and

2. $\varphi_{\text {oe }}: \mathcal{H}_{o}^{i}(L) \longrightarrow \mathcal{H}_{e}^{i+1}(L)$, the connecting homomorphism for the sequence $(7)$. 
Both operations are link invariants, because they arise as Bockstein homomorphisms associated to the short exact sequences of $\mathbb{Z}_{\xi}$-modules

$$
\begin{aligned}
& 0 \longrightarrow \mathbb{Z}_{o} \longrightarrow \mathbb{Z}_{\xi} \longrightarrow \mathbb{Z}_{e} \longrightarrow 0, \text { and } \\
& 0 \longrightarrow \mathbb{Z}_{e} \longrightarrow \mathbb{Z}_{\xi} \longrightarrow \mathbb{Z}_{e} \longrightarrow 0
\end{aligned}
$$

respectively, considered as coefficients for the unified Khovanov homology $\mathcal{C}_{\xi}(D)$. As in the case of Khovanov complexes, these sequences are consequences of the pullback description of $\mathbb{Z}_{\xi}$.

Tensoring the exact sequences (16) and (17) with $\mathbb{Z}_{2}$ reveals that $\varphi_{e o}$ and $\varphi_{o e}$ are integral lifts of Bockstein homomorphisms from the previous section.

Proposition 4.1. Given a link $L$, there are commuting squares
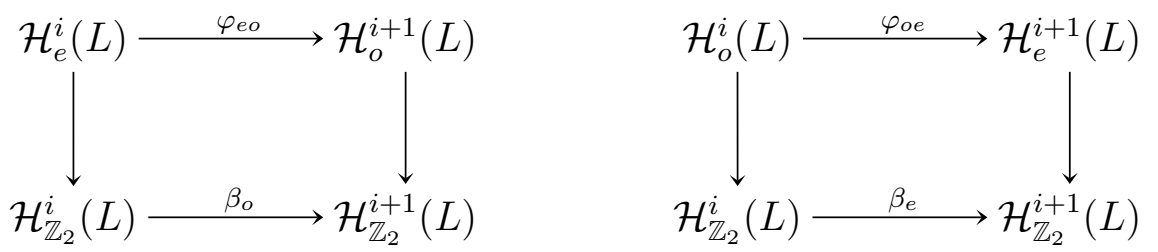

Proof. We start with finding a formula for $\varphi_{\text {eo }}$ using the pullback description of $\mathcal{C}_{\xi}(L)$. Pick a cocycle $x \in \mathcal{C}_{e}(L)$; it is covered by $(x, x) \in \mathcal{C}_{\xi}(L)$, where we identify the even and odd chain groups in the natural way. Then $d_{\xi}(x, x)=\left(0, d_{o} x\right)$ is the image of $\frac{1}{2} d_{o} x$, as the inclusion of the odd homology takes a chain $y$ into $(0,2 y)$. Notice that the division makes sense because modulo 2 we have $d_{o} x \equiv d_{e} x=0$. Hence, $\varphi_{e o}[x]=\left[\frac{1}{2} d_{o} x\right]$, which agrees modulo 2 with $\beta_{o}\left([x] \otimes \mathbb{Z}_{2}\right)$. The case of $\varphi_{o e}$ is proved likewise.

Corollary 4.2. Both $\varphi_{e o}$ and $\varphi_{o e}$ are annihilated by 2.

Proof. Since $\varphi_{e o}[x]=\left[\frac{1}{2} d_{o} x\right] \in \mathcal{H}_{o}(L)$, we immediately get that $\varphi_{e o}[2 x]=\left[d_{o} x\right]=0$. The case of $\varphi_{o e}$ is similar.

Combining Proposition 4.1 and Conjecture 3.8 results in an algebraic independence of integral operations.

Conjecture 4.3. All alternating compositions $\cdots \varphi_{e o} \varphi_{o e}$ and $\cdots \varphi_{o e} \varphi_{e o}$ are nontrivial.

Indeed, it is enough to find a link $L$ and a class $a \in \mathcal{H}_{o}(L)$ (resp. $\left.a \in \mathcal{H}_{e}(L)\right)$ such that the composition $\cdots \beta_{o} \beta_{e}$ (resp. $\cdots \beta_{e} \beta_{o}$ ) does not vanish on the $\mathbb{Z}_{2}$-reduction $\bar{a} \in \mathcal{H}_{\mathbb{Z}_{2}}(L)$. In particular, we know that

- both $\varphi_{e o} \varphi_{o e}$ and $\varphi_{o e} \varphi_{e o}$ are nonzero for $10_{124}=T_{3,5}$, and

- $\varphi_{e o} \varphi_{o e} \varphi_{e o}$ is nonzero for $T_{5,6}$.

We expect higher torus knots to provide examples for which longer compositions are nontrivial. 
The ring $\mathbb{Z}_{\xi}$ is the only nontrivial extension between $\mathbb{Z}_{e}$ and $\mathbb{Z}_{o}$. Indeed, free resolutions of $\mathbb{Z}_{e}$ and $\mathbb{Z}_{o}$ are given by infinite sequences

$$
\begin{aligned}
& 0 \longleftarrow \mathbb{Z}_{e} \longleftarrow \mathbb{Z}_{\xi} \stackrel{1-\xi}{\longleftarrow} \mathbb{Z}_{\xi} \stackrel{1+\xi}{\longleftarrow} \mathbb{Z}_{\xi} \longleftarrow \ldots \\
& 0 \longleftarrow \mathbb{Z}_{o} \longleftarrow \mathbb{Z}_{\xi} \stackrel{1+\xi}{\longleftarrow} \mathbb{Z}_{\xi} \stackrel{1-\xi}{\longleftarrow} \mathbb{Z}_{\xi} \longleftarrow \ldots
\end{aligned}
$$

from which one computes

$$
\begin{array}{ll}
\operatorname{Ext}_{\mathbb{Z}_{\xi}}^{1}\left(\mathbb{Z}_{e}, \mathbb{Z}_{e}\right) \cong 0, & \operatorname{Ext}_{\mathbb{Z}_{\xi}}^{1}\left(\mathbb{Z}_{e}, \mathbb{Z}_{o}\right) \cong \mathbb{Z}_{2}, \\
\operatorname{Ext}_{\mathbb{Z}_{\xi}}^{1}\left(\mathbb{Z}_{o}, \mathbb{Z}_{o}\right) \cong 0, & \operatorname{Ext}_{\mathbb{Z}_{\xi}}^{1}\left(\mathbb{Z}_{o}, \mathbb{Z}_{e}\right) \cong \mathbb{Z}_{2}
\end{array}
$$

Corollary 4.4. The operations $\varphi_{e o}$ and $\varphi_{o e}$ are the only nontrivial Bockstein-type operations between the even and odd Khovanov homology.

Similarly to the $\mathbb{Z}_{2}$ case, the operations $\varphi_{e o}$ and $\varphi_{o e}$ are primitive when regarded as operations acting on the direct sum of the even and odd Khovanov homology. Indeed, if we write $\mathcal{H}_{\oplus}(L):=\mathcal{H}_{e}(L) \oplus \mathcal{H}_{o}(L)$, then we can identify $\varphi_{e o}$ and $\varphi_{o e}$ with matrices

$$
\tilde{\varphi}_{e o}:=\left(\begin{array}{cc}
0 & 0 \\
\varphi_{e o} & 0
\end{array}\right) \quad \tilde{\varphi}_{o e}:=\left(\begin{array}{cc}
0 & \varphi_{o e} \\
0 & 0
\end{array}\right)
$$

respectively. Following the Künneth formula, we define for links $L$ and $L^{\prime}$

$$
\mathcal{H}_{e}(L) \hat{\otimes} \mathcal{H}_{e}\left(L^{\prime}\right):=\mathcal{H}_{e}(L) \otimes \mathcal{H}_{e}\left(L^{\prime}\right) \oplus \operatorname{Tor}\left(\mathcal{H}_{e}(L), \mathcal{H}_{e}\left(L^{\prime}\right)\right)[1] \cong \mathcal{H}_{e}\left(L \sqcup L^{\prime}\right)
$$

and likewise for $\mathcal{H}_{o}$ and $\mathcal{H}_{\oplus}$. Then $\mathcal{H}_{\oplus}\left(L \sqcup L^{\prime}\right) \cong \mathcal{H}_{e}(L) \hat{\otimes} \mathcal{H}_{e}\left(L^{\prime}\right) \oplus \mathcal{H}_{o}(L) \hat{\otimes} \mathcal{H}_{o}\left(L^{\prime}\right)$ is a direct summand of $\mathcal{H}_{\oplus}(L) \hat{\otimes} \mathcal{H}_{\oplus}\left(L^{\prime}\right)$, and the operations $\varphi_{e o}$ and $\varphi_{o e}$ regarded as endomorphisms of the product $\mathcal{H}_{\oplus}(L) \hat{\otimes} \mathcal{H}_{\oplus}\left(L^{\prime}\right)$ can be identified with matrices

$$
\begin{aligned}
& \tilde{\varphi}_{e o}=\left(\begin{array}{cccc}
0 & 0 & 0 & 0 \\
\mathrm{id} \hat{\otimes} \varphi_{e o} & 0 & 0 & 0 \\
\varphi_{e o} \hat{\otimes} \mathrm{id} & 0 & 0 & 0 \\
0 & \varphi_{e o} \hat{\otimes} \mathrm{id} & \mathrm{id} \hat{\otimes} \varphi_{e o} & 0
\end{array}\right)=\tilde{\varphi}_{e o} \hat{\otimes} \mathrm{id}_{\mathcal{H}_{\oplus}}+\mathrm{id}_{\mathcal{H}_{\oplus}} \hat{\otimes} \tilde{\varphi}_{e o}, \text { and } \\
& \tilde{\varphi}_{o e}=\left(\begin{array}{cccc}
0 & \mathrm{id} \hat{\otimes} \varphi_{o e} & \varphi_{o e} \hat{\otimes} \mathrm{id} & 0 \\
0 & 0 & 0 & \varphi_{o e} \hat{\otimes} \mathrm{id} \\
0 & 0 & 0 & \mathrm{id} \hat{\otimes} \varphi_{o e} \\
0 & 0 & 0 & 0
\end{array}\right)=\tilde{\varphi}_{o e} \hat{\otimes} \mathrm{id}_{\mathcal{H}_{\oplus}}+\mathrm{id}_{\mathcal{H}_{\oplus}} \hat{\otimes} \tilde{\varphi}_{o e},
\end{aligned}
$$

where we order the four summands of $\mathcal{H}_{\oplus}(L) \hat{\otimes} \mathcal{H}_{\oplus}\left(L^{\prime}\right)$ as even-even, even-odd, odd-even, and odd-odd. In particular, $\Phi:=\tilde{\varphi}_{e o}+\tilde{\varphi}_{o e}=\left(\begin{array}{cc}0 & \varphi_{o e} \\ \varphi_{e o} & 0\end{array}\right)$ is a $\sqcup$-primitive operation on $\mathcal{H}_{\oplus}(L)$. The same argument works for connected sums of links, showing the operations are \#-primitive as well. 
Remark 4.5. Using the primitivity as defined above, one may try to prove Conjecture 4.3 without referring to the $\mathbb{Z}_{2}$-operations. Unfortunately, $\Phi^{2^{r}}$ is again primitive for every $r>0$, due to the binomial formula (15) and Corollary 4.2.

\section{Reduced Khovanov homology}

Given a diagram $D$ of a knot $K$, choose a point $b$ on $D$ and consider the cobordism merging a circle to $D$ at the point $b$, as shown in Figure 3. This operation induces on the chain complex $\mathcal{C}_{\xi}(D)$ a module structure over $A_{\xi}=\mathbb{Z}_{\xi} v_{+} \oplus \mathbb{Z}_{\xi} v_{-}$, the algebra associated to a circle. This structure is independent of the chosen point $b$, see [Pu14].

Consider the subcomplex $\overline{\mathcal{C}}_{\xi}(D)$ of $\mathcal{C}_{\xi}(D)$ spanned by the chains annihilated by $v_{-}$. We call its homology the reduced unified homology $\overline{\mathcal{H}}_{\xi}(K)$ of $K$. A quick comparison with definitions of the reduced even and odd Khovanov homology [Kh04, ORS13] reveals that both are specializations of $\overline{\mathcal{H}}_{\xi}(K)$, cf. (2):

$$
\overline{\mathcal{H}}_{e}(K) \cong \overline{\mathcal{H}}_{\xi}\left(K ; \mathbb{Z}_{e}\right), \quad \overline{\mathcal{H}}_{o}(K) \cong \overline{\mathcal{H}}_{\xi}\left(K ; \mathbb{Z}_{o}\right) .
$$

In particular, there are reduced versions of all the homological operations defined so far:

- the reduced Bockstein operations $\bar{\beta}_{e}, \bar{\beta}_{o}: \overline{\mathcal{H}}_{\mathbb{Z}_{2}}^{i}(K) \longrightarrow \overline{\mathcal{H}}_{\mathbb{Z}_{2}}^{i+1}(K)$, and

- their integral lifts $\bar{\varphi}_{e o}: \overline{\mathcal{H}}_{e}^{i}(K) \longrightarrow \overline{\mathcal{H}}_{o}^{i+1}(K)$ and $\bar{\varphi}_{o e}: \overline{\mathcal{H}}_{o}^{i}(K) \longrightarrow \overline{\mathcal{H}}_{e}^{i+1}(K)$.

Clearly, the reduced homology is multiplicative with respect to the connected sum of knots, which implies that reductions of \#-primitive operations are primitive. In particular, all the reduced Bockstein homomorphisms are primitive. As before, we conjecture that the reduced Bockstein homomorphisms satisfy only the obvious relations.

Conjecture 5.1. The alternating compositions of reduced Bockstein homomorphisms are nonzero and different, and so are their integral lifts (given as alternating compositions of $\bar{\varphi}_{e o}$ and $\left.\bar{\varphi}_{o e}\right)$.

Example 5.2. The torus knot $T_{4,5}$ admits a class $u \in \overline{\mathcal{H}}_{\mathbb{Z}_{2}}\left(T_{4,5}\right)$, on which $\bar{\beta}_{e}$ vanishes but $\bar{\beta}_{e} \bar{\beta}_{o}$ does not, see Figure 4 .

\section{EXPERIMENTAL RESUlts}

We computed ranks of all the homological operations discussed in this paper in every bigrading for all prime knots with up to 16 crossings using KhoHo [Sh $\infty]$. We then compared these ranks for pairs of knots that have the same even and odd Khovanov homology. As discussed in the Introduction, the ranks of Bockstein homomorphisms $\beta_{e}$ and $\beta_{o}$ are fully determined by the even and odd Khovanov homology. Therefore, they do not provide any new information. However, the mixed Bockstein $\beta=\beta_{e}+\beta_{o}$ is different. It turns out that there exist 7 pairs of prime knots (14 if counted with mirror images) with up to 14 crossings 


\begin{tabular}{||l||l|l|l|l|l|l|l|l|l|l|l||}
\hline \hline$\overline{\mathcal{H}}_{e}$ & 0 & 1 & 2 & 3 & 4 & 5 & 6 & 7 & 8 & 9 & 10 \\
\hline \hline 28 & & & & & & & & & & & $\mathbb{Z}_{2}$ \\
\hline 26 & & & & & & & & & & $\mathbb{Z}$ & \\
\hline 24 & & & & & & & & $\mathbb{Z}$ & $\mathbb{Z}$ & & \\
\hline 22 & & & & & & $\mathbb{Z}$ & & $\mathbb{Z}_{2}$ & & & \\
\hline 20 & & & & & & & $\mathbb{Z}$ & & & & \\
\hline 18 & & & & $\mathbb{Z}$ & $\mathbb{Z}$ & & & & & & \\
\hline 16 & & & $\mathbb{Z}$ & & & & & & & & \\
\hline 14 & & & & & & & & & & & \\
\hline 12 & $\mathbb{Z}$ & & & & & & & & & & \\
\hline \hline
\end{tabular}

\begin{tabular}{||r||l|l|l|l|l|l|l|l|l|l|l||}
\hline \hline$\overline{\mathcal{H}}_{o}$ & 0 & 1 & 2 & 3 & 4 & 5 & 6 & 7 & 8 & 9 & 10 \\
\hline \hline 28 & & & & & & & & & & $\mathbb{Z}$ & $\mathbb{Z}$ \\
\hline 26 & & & & & & & & & & $\mathbb{Z}$ & \\
\hline 24 & & & & & & & & $\mathbb{Z}$ & $\mathbb{Z}$ & & \\
\hline 22 & & & & & & & $\mathbb{Z}_{2}$ & $\mathbb{Z}$ & & & \\
\hline 20 & & & & & & $\mathbb{Z}_{3}$ & $\mathbb{Z}$ & & & & \\
\hline 18 & & & & & $\mathbb{Z}_{2}$ & & & & & & \\
\hline 16 & & & $\mathbb{Z}$ & & & & & & & & \\
\hline 14 & & & & & & & & & & & \\
\hline 12 & $\mathbb{Z}$ & & & & & & & & & & \\
\hline \hline
\end{tabular}

\begin{tabular}{||r||c|c|c|c|c|c|c|c|c|c|c||}
\hline \hline$\overline{\mathcal{H}}_{\mathbb{Z}_{2}}$ & 0 & 1 & 2 & 3 & 4 & 5 & 6 & 7 & 8 & 9 & 10 \\
\hline \hline 28 & & & & & & & & & & $\mathbb{Z}_{2} \frac{\beta_{e}}{\mathbb{Z}_{2}}$ \\
\hline 26 & & & & & & & & & & $\mathbb{Z}_{2}$ & \\
\hline 24 & & & & & & & & $\mathbb{Z}_{2}$ & $\mathbb{Z}_{2}$ & & \\
\hline 22 & & & & & & $\mathbb{Z}_{2} \frac{\beta_{0}}{\longrightarrow} \mathbb{Z}_{2} \frac{\beta_{e}}{\mathbb{Z}_{2}}$ & & & \\
\hline 20 & & & & & & & $\mathbb{Z}_{2}$ & & & & \\
\hline 18 & & & & $\mathbb{Z}_{2} \frac{\beta_{0}}{\longrightarrow} \mathbb{Z}_{2}$ & & & & & & \\
\hline 16 & & & $\mathbb{Z}_{2}$ & & & & & & & & \\
\hline 14 & & & & & & & & & & \\
\hline 12 & $\mathbb{Z}_{2}$ & & & & & & & & & \\
\hline \hline
\end{tabular}

Figure 4. Even and odd reduced Khovanov homology as well as Bockstein homomorphisms on the reduced Khovanov homology over $\mathbb{Z}_{2}$ for the $\operatorname{knot} T_{4,5}$. 


\begin{tabular}{l|c|c|c} 
& $\leq 14$ crossings & $\leq 15$ crossings & $\leq 16$ crossings \\
\hline Number of knots (counting mirrors) & 67289 & 403348 & 2420670 \\
Pairs distinguished by ranks of $\beta$ & 14 & 122 & 1484 \\
Pairs distinguished by ranks of $\beta^{2}$ & 0 & 8 & 108 \\
Pairs distinguished by ranks of $\varphi_{o e}$ & 7 & 61 & 760 \\
Pairs distinguished by ranks of $\varphi_{e o}$ & 7 & 61 & 760 \\
Pairs distinguished by ranks of $\theta_{e}$ & 0 & 4 & 71 \\
Pairs distinguished by ranks of $\theta_{o}$ & 9 & 95 & 1232 \\
\hline
\end{tabular}

TABLE 3. Number of pairs of prime non-alternating knots with the same even and odd Khovanov homology that are distinguished by homological operations

that have the same even and odd Khovanov homology but different ranks of $\beta$. There are 61 (resp. 122) such pairs among knots with up to 15 crossings and 742 (resp. 1484) with up to 16 crossings, see Table 3 .

Corollary 6.1. The unified homology $\mathcal{H}_{\xi}(L)$ is a stronger link invariant than $\mathcal{H}_{e}(L) \oplus \mathcal{H}_{o}(L)$.

Here are the first 7 pairs of knots $^{2}$ distinguished by $\beta$ :

$$
\begin{array}{clrl}
13_{141}^{n} \leftrightarrow 14_{2551}^{n} & 13_{1002}^{n} \leftrightarrow 14_{6487}^{n} & 14_{1346}^{n} \leftrightarrow 14_{7711}^{n} \quad 14_{5293}^{n} \leftrightarrow 14_{12516}^{n} \\
14_{5373}^{n} \leftrightarrow 14_{12516}^{n} & 14_{6632}^{n} \leftrightarrow \overline{14}_{21021}^{n} & 14_{12393}^{n} \leftrightarrow 14_{12532}^{n} &
\end{array}
$$

Existence of such pairs can be explained by the observation that Bockstein homomorphisms are described by a noncanonical splitting $H^{i}\left(C, \mathbb{Z}_{2}\right) \cong H^{i}(C) \otimes \mathbb{Z}_{2} \oplus \operatorname{Tor}\left(H^{i+1}(C), \mathbb{Z}_{2}\right)$, and in case of Khovanov homology the two splittings, one for the even and one for the odd version, do not coincide. In other words, we cannot pick such isomorphisms for even and odd Khovanov homology that agree over $\mathbb{Z}_{2}$.

$\beta^{2}$ is, obviously, a much weaker invariant than $\beta \operatorname{since~} \operatorname{rk}\left(\beta^{2}\right) \leq \operatorname{rk} \beta$ (in an appropriate bigrading). Nonetheless, there are 4 (resp. 8) pairs of knots with 15 crossings that can be distinguished by $\beta^{2}$ :

$$
\begin{array}{ll}
15_{23106}^{n} \leftrightarrow 15_{56014}^{n} & 15_{23432}^{n} \leftrightarrow 15_{56014}^{n} \\
15_{44028}^{n} \leftrightarrow \overline{15}_{50224}^{n} & 15_{73047}^{n} \leftrightarrow \overline{15}_{91280}^{n}
\end{array}
$$

It is important to notice that the Khovanov homology modulo 2 for a knot $K$ and its mirror image $\bar{K}$ are dual to each other. As such, if any of the homological operations over $\mathbb{Z}_{2}$ has the same ranks for two knots, say, $K_{1}$ and $K_{2}$, then the ranks are the same for $\bar{K}_{1}$ and $\bar{K}_{2}$ as well. The situation is different for integral operations. It turns out that among

\footnotetext{
${ }^{2}$ Here, $13_{141}^{n}$ denotes the non-alternating knot number 141 with 13 crossings from the Knotscape knot table and $\overline{14}_{21021}^{n}$ is the mirror image of the knot $14_{21021}^{n}$.
} 
all knots with up to 14 crossings, both $\varphi_{o e}$ and $\varphi_{e o}$ distinguish 7 pairs of knots, but not their mirror images, cf. (28):

$$
\begin{aligned}
\overline{13}_{141}^{n} \leftrightarrow \overline{14}_{2551}^{n} & 13_{1002}^{n} \leftrightarrow 14_{6487}^{n} & 14_{1346}^{n} \leftrightarrow 14_{7711}^{n} & 14_{5293}^{n} \leftrightarrow 14_{12516}^{n} \\
14_{5373}^{n} \leftrightarrow 14_{12516}^{n} & 14_{6632}^{n} \leftrightarrow \overline{14}_{21021}^{n} & 14_{12393}^{n} \leftrightarrow 14_{12532}^{n} &
\end{aligned}
$$

One can consider integral homological operations of degree 2 as well: $\theta_{e}:=\varphi_{o e} \varphi_{e o}$ and $\theta_{o}:=\varphi_{e o} \varphi_{o e}$, the former defined for the even and the latter for the odd Khovanov homology. Both of them are, obviously, integral lifts of $\beta^{2}$, see (18). Computations reveal that $\theta_{o}$ distinguishes the same 7 pairs of knots with at most 14 crossings as in (30), plus two more:

$$
13_{651}^{n} \leftrightarrow 14_{16550}^{n} \quad 13_{661}^{n} \leftrightarrow 14_{16550}^{n}
$$

On the other hand, the first pair of knots with the same even and odd Khovanov homology but with different ranks of $\theta_{e}$ has 15 crossings, see Table 3 . There are 4 such pairs in total:

$$
\begin{array}{ll}
\overline{15}_{23106}^{n} \leftrightarrow \overline{15}_{56014}^{n} & \overline{15}_{23432}^{n} \leftrightarrow \overline{15}_{56014}^{n} \\
\overline{15}_{44028}^{n} \leftrightarrow 15_{50224}^{n} & \overline{15}_{73047}^{n} \leftrightarrow 15_{91280}^{n}
\end{array}
$$

Observation 6.2. For every pair of knots from (30) and (31), these knots are distinguished by the ranks of $\theta_{o}$, yet $\theta_{o}$ is trivial for their mirror images. This can be explained by noticing that a knot may be thin while its mirror image is not, see the case of $13_{1002}^{n}$ and $14_{6487}^{n}$ in Figure 5 .

Observation 6.3. Looking at Table 3, it appears that $\varphi_{e o}$ and $\varphi_{o e}$ are stronger invariants than $\beta$ and $\beta^{2}$, while $\theta_{o}$ is even stronger. This is indeed true with a few notable exceptions. Among all prime non-alternating knots with at most 16 crossings, there are two pairs that can be distinguished by the rank of $\beta$ but not by either $\varphi_{e o}$ or $\varphi_{o e}$ :

$$
16_{129312}^{n} \leftrightarrow \overline{16}_{640105}^{n} \quad 16_{240722}^{n} \leftrightarrow 16_{640105}^{n}
$$

Also, there are two pairs of knots that can be distinguished by $\varphi_{e o}$ and $\varphi_{o e}$, but not by $\theta_{o}$ :

$$
\overline{16}_{198481}^{n} \leftrightarrow \overline{16}_{416282}^{n} \quad \overline{16}_{639703}^{n} \leftrightarrow \overline{16}_{698630}^{n}
$$

On the other hand, the lists of distinguishable knots with up to 16 crossings for $\varphi_{e o}$ and $\varphi_{o e}$ indeed coincide.

We also looked at all the knots that have the same even and odd reduced Khovanov homology, but different ranks of the reduced homological operations. Since the width of the reduced homology is always less by 1 than the width of the corresponding unreduced homology, and since the even reduced Khovanov homology has, in general, very little torsion, it is natural to expect that the reduced homological operations would be not as strong as their unreduced counterparts. This is indeed the case. 


\begin{tabular}{||r||l|l|l|l|l|l|l|l|l|l|l||}
\hline \hline $\mathcal{H}_{o}$ & -6 & -5 & -4 & -3 & -2 & -1 & 0 & 1 & 2 & 3 & 4 \\
\hline \hline 11 & & & & & & & & & & & $\mathbb{Z}$ \\
\hline 9 & & & & & & & & & & $\mathbb{Z}^{2}$ & $\mathbb{Z}$ \\
\hline 7 & & & & & & & & & $\mathbb{Z}^{4}$ & $\mathbb{Z}^{2}$ & \\
\hline 5 & & & & & & & & $\mathbb{Z}^{5}$ & $\mathbb{Z}^{4} \oplus \mathbb{Z}_{2}$ & & \\
\hline 3 & & & & & & & $\mathbb{Z}^{6-}$ & $\mathbb{Z}^{5} \oplus \mathbb{Z}_{2}$ & $\mathbb{Z}_{2}$ & & \\
\hline 1 & & & & & & $\mathbb{Z}^{7}$ & $\mathbb{Z}^{6} \oplus \mathbb{Z}_{2}$ & $\mathbb{Z}_{2}$ & & & \\
\hline-1 & & & & & $\mathbb{Z}^{6}$ & $\mathbb{Z}^{7} \oplus \mathbb{Z}_{2}$ & $\mathbb{Z}_{2}$ & & & & \\
\hline-3 & & & & $\mathbb{Z}^{5}$ & $\mathbb{Z}^{6} \oplus \mathbb{Z}_{2}$ & $\mathbb{Z}_{2}$ & & & & & \\
\hline-5 & & & $\mathbb{Z}^{4}$ & $\mathbb{Z}^{5}$ & $\mathbb{Z}_{2}$ & & & & & & \\
\hline-7 & & $\mathbb{Z}^{2}$ & $\mathbb{Z}^{4}$ & & & & & & & & \\
\hline-9 & $\mathbb{Z}$ & $\mathbb{Z}^{2}$ & & & & & & & & & \\
\hline-11 & $\mathbb{Z}$ & & & & & & & & & & \\
\hline \hline
\end{tabular}

\begin{tabular}{|c|c|c|c|c|c|c|c|c|c|c|c|}
\hline $\mathcal{H}_{o}$ & -4 & -3 & -2 & -1 & 0 & 1 & 2 & 3 & 4 & 5 & 6 \\
\hline 11 & & & & & & & & & & & $\mathbb{Z}$ \\
\hline 9 & & & & & & & & & & $\mathbb{Z}^{2}$ & $\mathbb{Z}$ \\
\hline 7 & & & & & & & & & $\mathbb{Z}^{4}$ & $\mathbb{Z}^{2}$ & \\
\hline 5 & & & & & & & & $\mathbb{Z}^{5} \oplus \mathbb{Z}_{2}$ & $\mathbb{Z}^{4}$ & & \\
\hline 3 & & & & & & & $\mathbb{Z}^{6} \oplus \mathbb{Z}_{2}$ & $\mathbb{Z}^{2} \oplus \mathbb{Z}_{2}$ & & & \\
\hline 1 & & & & & & $\mathbb{Z}^{7} \oplus \mathbb{Z}_{2}$ & $\mathbb{Z}^{6} \oplus \mathbb{Z}_{2}$ & & & & \\
\hline-1 & & & & & $\mathbb{Z}^{6} \oplus \mathbb{Z}_{2}$ & $\mathbb{Z}^{7} \oplus \mathbb{Z}_{2}$ & & & & & \\
\hline-3 & & & & $\mathbb{Z}^{5} \oplus \mathbb{Z}_{2}$ & $\mathbb{Z}^{6} \oplus \mathbb{Z}_{2}$ & & & & & & \\
\hline-5 & & & $\mathbb{Z}^{4}$ & $\mathbb{Z}^{5} \oplus \mathbb{Z}_{2}$ & & & & & & & \\
\hline-7 & & $\mathbb{Z}^{2}$ & $\mathbb{Z}^{4}$ & & & & & & & & \\
\hline-9 & $\mathbb{Z}$ & $\mathbb{Z}^{2}$ & & & & & & & & & \\
\hline-11 & $\mathbb{Z}$ & & & & & & & & & & \\
\hline
\end{tabular}

FiguRE 5. Odd Khovanov homology for knots $13_{1002}^{n}$ and $14_{6487}^{n}$ (upper table) and their mirror images (lower table). The solid blue arrows indicate places where the operation $\theta_{o}$ is surjective for both knots. At the dashed red arrow, $\theta_{o}$ is surjective for $13_{1002}^{n}$, but is a zero map for $14_{6487}^{n}$. Notice that there is no place for a nontrivial $\theta_{o}$ in the lower table. 


\begin{tabular}{|c|c|c|c|c|c|c|c|c|c|c|c|c|c|c|c|c|}
\hline$\overline{\mathcal{H}}_{o}$ & -8 & -7 & -6 & -5 & -4 & -3 & -2 & -1 & 0 & 1 & 2 & 3 & 4 & 5 & 6 & 7 \\
\hline 10 & & & & & & & & & & & & & & & & $\mathbb{Z}$ \\
\hline 8 & & & & & & & & & & & & & & & $\mathbb{Z}^{2}$ & \\
\hline 6 & & & & & & & & & & & & & & $\mathbb{Z}^{2}$ & & \\
\hline 4 & & & & & & & & & & & & & $\mathbb{Z}^{2} \oplus \mathbb{Z}_{2}$ & & & \\
\hline 2 & & & & & & & & & & & $\mathbb{Z}$ & $H_{1}$ & & & & \\
\hline 0 & & & & & & & & & & $\mathbb{Z}_{2}$ & $\mathbb{Z}_{2}^{2} \oplus \mathbb{Z}_{3}^{2}$ & & & & & \\
\hline-2 & & & & & & & & $\mathbb{Z}^{-}$ & $\bar{H}_{1}$ & $\mathbb{Z}_{2}^{2} \oplus \mathbb{Z}_{3}$ & & & & & & \\
\hline-4 & & & & & & & $\mathbb{Z}^{2}$ & $\mathrm{H}_{2}$ & $\mathbb{Z}_{2}$ & & & & & & & \\
\hline-6 & & & & & & $\mathbb{Z}^{3}$ & $H_{2}$ & & & & & & & & & \\
\hline-8 & & & & & $\mathbb{Z}^{4}$ & $\mathbb{Z}^{2} \oplus \mathbb{Z}_{2}^{2}$ & & & & & & & & & & \\
\hline-10 & & & & $\mathbb{Z}^{4}$ & $\mathbb{Z} \oplus \mathbb{Z}_{2}$ & & & & & & & & & & & \\
\hline-12 & & & $\mathbb{Z}^{4}$ & & & & & & & & & & & & & \\
\hline-14 & & $\mathbb{Z}^{3}$ & & & & & & & & \multirow{2}{*}{\multicolumn{7}{|c|}{ Notation: $H_{n}:=\mathbb{Z}^{n} \oplus \mathbb{Z}_{2}^{2} \oplus \mathbb{Z}_{3}$}} \\
\hline-16 & $\mathbb{Z}$ & & & & & & & & & & & & & & & \\
\hline
\end{tabular}

\begin{tabular}{|c|c|c|c|c|c|c|c|c|c|c|c|c|c|c|c|c|}
\hline$\overline{\mathcal{H}}_{o}$ & -7 & -6 & -5 & -4 & -3 & -2 & -1 & 0 & 1 & 2 & 3 & 4 & 5 & 6 & 7 & 8 \\
\hline 16 & & & & & & & & & & & & & & & & $\mathbb{Z}$ \\
\hline 14 & & & & & & & & & & & & & & & $\mathbb{Z}^{3}$ & \\
\hline 12 & & & & & & & & & & & & & & $\mathbb{Z}^{4}$ & & \\
\hline 10 & & & & & & & & & & & & $\mathbb{Z}$ & $\mathbb{Z}^{4} \oplus \mathbb{Z}_{2}$ & & & \\
\hline 8 & & & & & & & & & & & $\mathbb{Z}^{2}$ & $\mathbb{Z}^{4} \oplus \mathbb{Z}_{2}^{2}$ & & & & \\
\hline 6 & & & & & & & & & & $\mathbb{Z}^{2}$ & $H_{3}$ & & & & & \\
\hline 4 & & & & & & & & & $\mathbb{Z}^{2} \oplus \mathbb{Z}_{2}$ & $\mathrm{H}_{2}$ & & & & & & \\
\hline 2 & & & & & & & & $H_{1}$ & $H_{1}$ & & & & & & & \\
\hline 0 & & & & & & & $\mathbb{Z}_{2}^{2} \oplus \mathbb{Z}_{3}^{2}$ & $\mathbb{Z}_{2}$ & & & & & & & & \\
\hline-2 & & & & & $\mathbb{Z}$ & $H_{1}$ & & & & & & & & & & \\
\hline-4 & & & & $\mathbb{Z}^{2}$ & $\mathbb{Z}_{2}$ & & & & & & & & & & & \\
\hline-6 & & & $\mathbb{Z}^{2}$ & & & & & & & & & & & & & \\
\hline-8 & & $\mathbb{Z}^{2}$ & & & & & & & & \multirow{2}{*}{\multicolumn{7}{|c|}{ Notation: $H_{n}:=\mathbb{Z}^{n} \oplus \mathbb{Z}_{2}^{2} \oplus \mathbb{Z}_{3}$}} \\
\hline-10 & $\mathbb{Z}$ & & & & & & & & & & & & & & & \\
\hline
\end{tabular}

Figure 6. Odd reduced Khovanov homology for knots $16_{235548}^{n}$ and $16_{635483}^{n}$ (upper table) and their mirror images (lower table). The solid blue arrow indicate a place where the operation $\theta_{o}$ is surjective for both knots. At the dashed red arrow, $\theta_{o}$ is non-trivial for $16_{235548}^{n}$, but is a zero map for $16_{635483}^{n}$. Notice that there is no place for a nontrivial $\theta_{o}$ in the lower table. 
Observation 6.4. Among all prime non-alternating knots with at most 16 crossings that have the same even and odd reduced Khovanov homology, there are only 4 pairs that can be distinguished by ranks of either $\bar{\beta}$, or $\bar{\varphi}_{o e}$, or $\bar{\varphi}_{e o}$, or $\bar{\theta}_{o}$ :

$$
\begin{array}{lll}
\overline{16}_{209296}^{n} \leftrightarrow \overline{16}_{699643}^{n} & 16_{235548}^{n} \leftrightarrow 16_{635483}^{n} \\
\overline{16}_{485898}^{n} \leftrightarrow 16_{543682}^{n} & \overline{16}_{910482}^{n} \leftrightarrow 16_{919988}^{n}
\end{array}
$$

$\bar{\beta}$ distinguishes their mirror images as well, but no other reduced homological operation does. See Figure 6 for an example.

We finish this paper with a few more conjectures of various levels of plausibility.

Conjecture 6.5. Every two knots that are distinguished by the ranks of $\varphi_{e o}$ are also distinguished by those of $\varphi_{o e}$ and vise versa. The same is also true for $\bar{\varphi}_{e o}$ and $\bar{\varphi}_{o e}$.

Conjecture 6.6. $\mathcal{H}_{\xi}(L)$ is even stronger invariant than ranks of all the homological operations discussed in this paper.

\section{REFERENCES}

[Ha10] A. Hatcher, Algebraic Topology, Cambridge Univ. Press, 2010.

[Kh99] M. Khovanov, A categorification of the Jones polynomial, Duke Math. J. 101 (2000), no. 3, 359-426. E-print: arXiv:math/9908171

[Kh04] M. Khovanov, Patters in Knot Cohomology, I, Experiment. Math. 12(3):365-374, 2003. E-print: arXiv:math/0201306

[LS12] R. Lipshitz, S. Sarkar, A Steenrod Square on Khovanov Homology, to appear in the Journal of Topology. E-print: arXiv:1204.5776

[ORS13] P. Ozsváth, J. Rasmussen, Z. Szabó, Odd Khovanov homology, Alg. Geom. Top. 13:1465-1488, 2013. E-print: arXiv:0710.4300.

[Pu08] K. K. Putyra, Cobordisms with chronologies and a generalized Khovanov complex, Masters' Thesis, Jagiellonian University, 2008. E-print: arXiv:1004.0889.

[Pu13] K. K. Putyra, A 2-category of chronological cobordisms and odd Khovanov homology, Banach Center Publ 203:287-351, 2014. E-print: arXiv:1310.1895v2.

[Pu14] K. K. Putyra, On an triply graded Khovanov homology, to appear in JKTR, 2016. E-print: arXiv:1501.05293.

[Ro76] D. Rolfsen, Knots and links, AMS Chelsea Pub., 1976.

[Sh11] A. Shumakovitch, Patterns in odd Khovanov homology, J. Knot Theory Ramifications, 20:203-222, 2011. E-print: arXiv:1101.5607.

[Sh $\infty$ ] A. Shumakovitch, KhoHo - a program for computing and studying Khovanov homology, https://github.com/AShumakovitch/KhoHo

ETH Zürich, Institute for Theoretical Studies, Zürich, Switzerland

E-mail address: krzysztof.putyra@eth-its.ethz.ch

Department of Mathematics, The George Washington University, Washington DC, U.S.A. E-mail address: Shurik@gwu.edu 